\title{
Contraction of Dynamically Masked Deep Neural Networks for Efficient Video Processing
}

\author{
Rueckauer, Bodo ; Liu, Shih-Chii
}

\begin{abstract}
Sequential data such as video are characterized by spatio-temporal redundancies. As of yet, few deep learning algorithms exploit them to decrease the often massive cost during inference. This work leverages correlations in video data to reduce the size and run-time cost of deep neural networks. Drawing upon the simplicity of the typically used ReLU activation function, we replace this function by dynamically updating masks. The resulting network is a simple chain of matrix multiplications and bias additions, which can be contracted into a single weight matrix and bias vector. Inference then reduces to an affine transformation of the input sample with these contracted parameters. We show that the method is akin to approximating the neural network with a first-order Taylor expansion around a dynamically updating reference point. For triggering these updates, one static and three data-driven mechanisms are analyzed. We evaluate the proposed algorithm on a range of tasks, including pose estimation on surveillance data, road detection on KITTI driving scenes, object detection on ImageNet videos, as well as denoising MNIST digits, and obtain compression rates up to $3.6 \times$.
\end{abstract}

DOI: https://doi.org/10.1109/tcsvt.2021.3066241

Posted at the Zurich Open Repository and Archive, University of Zurich

ZORA URL: https://doi.org/10.5167/uzh-211983

Journal Article

Accepted Version

Originally published at:

Rueckauer, Bodo; Liu, Shih-Chii (2022). Contraction of Dynamically Masked Deep Neural Networks for Efficient Video Processing. IEEE Transactions on Circuits and Systems for Video Technology, 32(2):621633.

DOI: https://doi.org/10.1109/tcsvt.2021.3066241 


\title{
Contraction of Dynamically Masked Deep Neural Networks for Efficient Video Processing
}

\author{
Bodo Rueckauer and Shih-Chii Liu, Senior Member, IEEE
}

\begin{abstract}
Sequential data such as video are characterized by spatio-temporal redundancies. As of yet, few deep learning algorithms exploit them to decrease the often massive cost during inference. This work leverages correlations in video data to reduce the size and run-time cost of deep neural networks. Drawing upon the simplicity of the typically used ReLU activation function, we replace this function by dynamically updating masks. The resulting network is a simple chain of matrix multiplications and bias additions, which can be contracted into a single weight matrix and bias vector. Inference then reduces to an affine transformation of the input sample with these contracted parameters. We show that the method is akin to approximating the neural network with a first-order Taylor expansion around a dynamically updating reference point. For triggering these updates, one static and three data-driven mechanisms are analyzed. We evaluate the proposed algorithm on a range of tasks, including pose estimation on surveillance data, road detection on KITTI driving scenes, object detection on ImageNet videos, as well as denoising MNIST digits, and obtain compression rates up to $3.6 \times$.
\end{abstract}

Index Terms-Deep neural networks, network compression, Taylor approximation, masking

\section{INTRODUCTION}

Natural video is often characterized by local spatio-temporal redundancies between frames, e.g. in surveillance or freeway driving recordings. Deep Neural Networks (DNNs) are powerful tools to process image data, but can be prohibitively expensive for use in mobile devices or always-on scenarios. The high degree of correlation between consecutive samples in sequential data calls for exploitation in DNNs.

To capitalize on this correlation for a more efficient solution to video processing, we previously presented a method to linearize part of a DNN or an entire DNN around a dynamically updating reference point. This dynamic updating is similar to the evaluation of a first-order Taylor expansion of a network function around an operating point [1]. By combining the linearized parts of the DNN, we arrive at a compressed DNN which has fewer neurons and parameters, and therefore also reduced operations during inference.

This network approximation method draws upon two common characteristics of DNNs: 1) The composite structure of neural networks where the mathematical function of a set of layers consists of a sequence of matrix multiplications. 2) The piecewise linear nature of the frequently used Rectifying Linear Unit (ReLU) activation function.

By replacing the element-wise activation function with a mask vector, a stack of layers can be contracted into a

B. Rueckauer and S.-C. Liu are with the Institute of Neuroinformatics, University of Zurich and ETH Zurich, Winterthurerstrasse 190, 8057 Zurich, Switzerland. E-mail: rbodo@ini.uzh.ch single layer. This layer, represented by a single weight matrix, approximates the function of the original layer stack around a reference point. The reference point, along with the contracted weight matrix, can be updated when temporal changes in the input sequence become large. These updates are expected to be sparse in slowly varying scenes. In between updates, inference is done cheaply using the contracted weight matrix.

In [1], we apply this compression method to a denoising autoencoder and demonstrate little loss in accuracy but savings in computation. This paper extends the previous work in three ways. First, we evaluate an improved criterion for dynamically updating the activation masks. Second, we discuss in detail the algorithm implementation and the computational cost. Finally, we validate the findings in [1] by applying this method to a diverse set of tasks, namely, a fully-convolutional network for semantic segmentation on the KITTI driving benchmark [2], pose estimation on surveillance data, and the YOLO architecture [3] for object detection on KITTI and a subset of the ImageNet object-detection-from-video challenge [4].

\section{A. Related Work}

1) Network Compression: Various methods have been proposed for making DNNs more efficient in processing sequential data by drawing upon redundancies in the input stream. For example, the authors of the Skip RNN [5] employ a control variable for each neuron that is used during training. This variable determines whether the state of the neuron is updated or copied over from the previous time step. A regularization term encourages the model to use a reduced number of state updates during training. This update skipping can be seen as a zero-order approximation: The state is kept constant (copied over). In contrast, our method replaces nonlinearities with masks and because changes can still be propagated through these masks, our method gives a first-order approximation.

Delta RNNs [6] reduce the computation in the network by capitalizing on the stability of RNN activation patterns and transmitting neuron activations only when the change in activation across two sequential timesteps exceeds a defined threshold. Change-based Convolutional Neural Networks (CNNs) [7] are based on a similar principle by exploiting the spatio-temporal sparsity of pixel changes in video data. The resulting accuracy drop from skipping computations is proportional to the targeted efficiency gain and the difficulty of the dataset. Unlike [7], our method does not discard small activation changes via a local threshold, instead all changes are propagated through the mask that replaces the nonlinearity.

Other compression methods include the use of low-precision models [8]-[10], pruning of redundant weights, feature maps 
or entire layers [11]-[13], and training convolutional architectures with fewer connections [14], [15]. Others train networks to develop input-dependent paths within the DNN [16]. In our case, network parts are not skipped or removed, but layer stacks are contracted to a single layer by replacing the ReLU nonlinearities with a dynamic mask. A comprehensive review of algorithm and hardware optimizations for more efficient DNN processing is given in [17].

2) Integrating Temporal Information: [18] presented a video recognition framework that runs a DNN on certain key frames and propagates the feature maps corresponding to a key frame to subsequent frames via a flow field. The optical flow is computed with a CNN that is trained end-to-end with the video recognition model. The method is similar to ours in the use of key frames, but differs in how inference is done between key frames (propagating hidden layer states along flow vectors versus linearizing the network function and contracting a stack of layers). The key frames in [18] are updated at regular intervals, while we propose various inputdriven update predictors. Lastly, their method makes explicit use of the temporal correlation in a video via optical flow whereas we implicitly draw on this assumption to motivate a linear approximation.

Another way to integrate temporal coherence for object detection in video has been proposed by [19]. Based on the assumption that objects obey constraints of physical continuity, the authors apply a post-processing step to the proposed bounding boxes to form so-called tubelets, i. e. bounding boxes linked across frames. By thus propagating detection results across frames and revising detection confidences based on the tubelet context, the detection accuracy is improved. This approach (which focuses on accuracy) could be combined with our work (which focuses on efficiency), to improve the mask vector used during layer contraction.

[20] improve vehicle detection in surveillance videos by adding a memory block to encode the temporal context of extracted CNN features. Since their approach adds a module onto an exchangeable CNN backbone, it can be combined seamlessly with our method, which operates purely on the CNN base model.

3) Taylor Expansion in DNNs: We show in Sec. II that our network contraction method can be seen as a first order Taylor expansion, where the derivatives are taken with respect to the network input. Taylor expansions are also applied to neural networks in other contexts, e. g. to explain nonlinear classification decisions [21], to generate a class saliency map of a specific input [22], and to analyze the learning convergence under various optimizers [23].

4) Activation Patterns and Linear Regions in DNNs: The piecewise linear nature of ReLU-DNNs gives rise to linear regions in the input space, where the gradient of the loss with respect to the input can be shown to be stable, leading to robustness against adversarial attacks [24]. These linear regions are defined by activation patterns [25], i.e. binary masks that encode the on/off state of the ReLU for each neuron in the network. These linear activation patterns have been studied in the context of neuron visualization [26], of estimating a network's expressiveness by counting the number of linear regions [25], [27], [28], and in adversarial attacks [26], [29], [30]. To the best of our knowledge, this is the first work which pursues computational savings by making use of static activation patterns in linear regions of the input space.

\section{MethodS}

In Sec. II-A we review the masking and contraction method as proposed in previous work [1], and extend it with an improved dynamic indicator for mask updates (Sec. II-B). We describe the algorithm implementation in Sec. II-C and discuss the reduction in the computational cost in Sec. II-D.

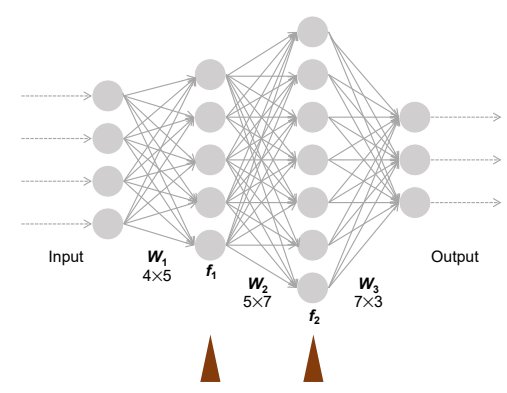

(a)

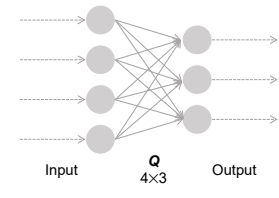

(b)
Fig. 1. Illustration of network masking and contraction. (a) Activation functions are first replaced by dynamically updating masks (triangles, cf. Eq. 3). (b) Hidden layer weight and mask matrices are then contracted (Eq. 5). Adapted from [1].

\section{A. Activation Masking and Network Contraction}

In this work, we consider feed-forward deep neural networks. Each neuron in a network layer receives as input the linear combination of the outputs of the preceding layer, and applies a nonlinear activation function on this weighted sum of inputs. For instance, an $n$-layer fully-connected Artificial Neural Network (ANN) has the following computational structure:

$$
\begin{array}{r}
F(\mathbf{x})=W^{n} f\left(W^{n-1} \ldots f\left(W^{1} \mathbf{x}+\mathbf{b}^{1}\right)+\right. \\
\left.\cdots+\mathbf{b}^{n-1}\right)+\mathbf{b}^{n},
\end{array}
$$

where $F$ is the network output, $\mathbf{x}$ is the network input, pairs $\left(W^{k}, \mathbf{b}^{k}\right)$ represent the weights and biases of layer $k \in[1, n]$, and $f$ is the nonlinear activation function of a neuron. Typically, the network output is also passed through a final nonlinearity $f_{\text {out }}$, e. g. a softmax in a classification task. To simplify notation in the following equations, we do not explicitly write this output nonlinearity, but imply that it is applied as usual after a forward-pass through the network. A common nonlinearity for hidden layers is the ReLU, which is a special case of the leaky ReLU:

$$
f\left(z_{i}^{k}\right)=\left\{\begin{aligned}
z_{i}^{k} & \text { if } z_{i}^{k}>0 \\
\alpha z_{i}^{k} & \text { otherwise }
\end{aligned}\right.
$$

The variable $z_{i}^{k}$ stands for the pre-activation of neuron $i$ in layer $k$, i.e. the summed output before applying the nonlinearity. The leak parameter, $\alpha$, defines the slope of the 
left branch and typically has a small value (e.g. $\alpha=0.1$ ). In the case of the standard ReLU, $\alpha=0$.

From (2), it is apparent that the element-wise application of the (leaky) ReLU $f$ in (1) is equivalent to the element-wise multiplication with a mask $\mathbf{m}^{k}$ :

$$
\begin{array}{r}
F(\mathbf{x})=W^{n} \mathbf{m}^{n-1} \odot\left(W^{n-1} \ldots \mathbf{m}^{1} \odot\left(W^{1} \mathbf{x}+\mathbf{b}^{1}\right)+\right. \\
\left.\cdots+\mathbf{b}^{n-1}\right)+\mathbf{b}^{n}
\end{array}
$$

where $\odot$ denotes the Hadamard product. In this work, the Hadamard product takes precedence over the matrixmultiplications, i.e. the mask $\mathbf{m}^{k}$ is applied to the preactivation of layer $k$ before multiplication with the weights $W^{k+1}$ of layer $k+1$. The mask $\mathbf{m}^{k}$ of layer $k$ is a vector whose length is equal to the number of neurons in layer $k$. Its entries are defined by ${ }^{1}$

$$
m_{i}^{k}= \begin{cases}1 & \text { if } z_{i}^{k}>0 \\ \alpha & \text { else. }\end{cases}
$$

Because matrix multiplications are associative, we can contract any number of matrix-, mask-, and vector-products in (3) into a single weight matrix $Q$ and bias vector $\mathbf{q}$ (see also Fig. 1a):

$$
\begin{aligned}
& F(\mathbf{x})=\left.Q\right|_{\mathbf{x}} \mathbf{x}+\left.\mathbf{q}\right|_{\mathbf{x}}, \text { where } \\
& \left.Q\right|_{\mathbf{x}}=W^{n} \operatorname{diag}\left(\mathbf{m}^{n-1}\right) \odot W^{n-1} \ldots \operatorname{diag}\left(\mathbf{m}^{1}\right) \odot W^{1}, \\
& \left.\mathbf{q}\right|_{\mathbf{x}}=W^{n} \mathbf{m}^{n-1} \odot\left(W^{n-1} \ldots \mathbf{m}^{1} \odot \mathbf{b}^{1}+\right. \\
& \left.\ldots+\mathbf{b}^{n-1}\right)+\mathbf{b}^{n} .
\end{aligned}
$$

These expressions are derived by expanding the nested products in (3). The mask vector is written in matrix-diagonal form so all multiplicands in $Q$ are matrices.

The key idea to reduce the run-time cost of a neural network on sequential data is to use a sample $\mathbf{x}_{*}$ to compute the $\left.(Q, \mathbf{q})\right|_{\mathbf{x}_{*}}$ parameters once, and then to perform inference on the next few samples in the sequence by using the much simplified affine transformation (5).

This process is related to the linearization of a complicated function (in our case the network $F(\mathbf{x})$ ) via Taylor expansion around a reference point $\mathbf{x}_{*}$ :

$$
T_{F}(\mathbf{x})=F\left(\mathbf{x}_{*}\right)+\left.J_{F}\right|_{\mathbf{x}_{*}}\left(\mathbf{x}-\mathbf{x}_{*}\right)+\ldots,
$$

with $\left.J_{F}\right|_{\mathbf{x}_{*}}=\left.\frac{\partial F}{\partial \mathbf{x}}\right|_{\mathbf{x}_{*}}$ the Jacobian of $F$ evaluated at $\mathbf{x}_{*}$. We determine the conditions under which our contracted network $C_{F}(\mathrm{x})$, defined by

$$
C_{F}(\mathrm{x})=\left.Q\right|_{\mathbf{x}_{*}} \mathbf{x}+\left.\mathbf{q}\right|_{\mathbf{x}_{*}},
$$

resembles a Taylor approximation around the reference point $\mathbf{x}_{*}$. By construction (c.f. (1)), the contracted network exactly equals the original network at the reference point:

$$
C_{F}\left(\mathrm{x}_{*}\right)=\left.Q\right|_{\mathbf{x}_{*}} \mathbf{x}_{*}+\left.\mathbf{q}\right|_{\mathbf{x}_{*}}=F\left(\mathbf{x}_{*}\right)
$$

\footnotetext{
${ }^{1}$ Because the mask depends on the current input sample, it could be written as $\left.\mathbf{m}^{k}\right|_{\mathbf{x}}$. We omit this subscript here for clarity and introduce it later.
}

By using identity (9) to replace the zero-order term in (7), and after some rearranging, we obtain:

$$
T_{F}(\mathbf{x})=\left.\mathbf{q}\right|_{\mathbf{x}_{*}}+\left(\left.Q\right|_{\mathbf{x}_{*}}-\left.J_{F}\right|_{\mathbf{x}_{*}}\right) \mathbf{x}_{*}+\left.J_{F}\right|_{\mathbf{x}_{*}} \mathbf{x}+\ldots
$$

Comparing coefficients of (8) and (10), we can see that the contracted network is identical to a first-order Taylor expansion if $Q$ is equal to the Jacobian:

$$
C_{F}(\mathbf{x})=T_{F}^{(1)}(\mathbf{x}) \quad \text { if }\left.\quad Q\right|_{\mathbf{x}_{*}}=\left.J_{F}\right|_{\mathbf{x}_{*}} .
$$

In Sec. III, we show that this relation holds through our experiments with a denoising autoencoder.

Having established a relationship between the contracted network and the first-order Taylor approximation of the original network, we now consider when to update the $Q$ matrix at a new reference point $\mathbf{x}_{*}$. At $\mathbf{x}_{*}$, the simplified affine transformation (5) is exactly equivalent to a standard forwardpass through the original multi-layer network. As the input samples begin to deviate from the reference point, the masks m used for computing the contracted $\left.(Q, \mathbf{q})\right|_{\mathbf{x}_{*}}$ parameters remain accurate only under the condition that the neurons do not change the sign of their activity $\mathbf{z}$ (c. f. (4)). For instance, if a neuron $i$ in layer $k$ changes from being positively activated at sample $\mathbf{x}_{*}=\mathbf{x}_{t}$ to being negatively activated at sample $\mathbf{x}_{t+1}$, then the corresponding entry in the mask vector $m_{i}^{k}$ should be $\alpha$ instead of 1 , and the $\left.(Q, \mathbf{q})\right|_{\mathbf{x}_{t}}$ parameters introduce an error. Note that such errors occur exclusively at sign changes: Otherwise, the $\left.(Q, \mathbf{q})\right|_{\mathbf{x}_{t}}$ parameters accurately encode the network function even under arbitrarily large changes of $z_{i}^{k}$.

To keep the network operation accurate even when activation signs are changing over the course of presenting an input sequence, it is necessary to recompute the $(Q, \mathbf{q})$ parameters at appropriate intervals, using updated binary masks that represent the state of network activations at the new reference point. Thus, we face an accuracy-efficiency trade-off: The less often the update of the $(Q, \mathbf{q})$ parameters, the lower is the runtime cost of inference, but the higher is the risk of inaccurate activation masks. This trade-off is discussed in Section III. We now turn to the question of how mask updates can be predicted.

\section{B. Indicators for Mask Updates}

The masks $\mathbf{m}^{k}$ needed to compute $(Q, \mathbf{q})$ depend on the current input $\mathrm{x}$ and the resulting activity of intermediate layers. We describe in more detail the three update indicators used in previous work [1], and propose a fourth metric which directly measures the number of activation sign changes.

1) Regular Frame Update: The most straight-forward baseline criterion for mask updates is the use of a regular update interval. The hyperparameter in this case is to perform the network contraction after every $n$-th frame (Fig. 2a). The advantages of this criterion are that it is easy to interpret and relate to expected savings in computations. Further, it offers a safety guarantee by limiting the longest period without updates to $n$ frames. On the other hand, potential savings due to rather static scenes with more than $n$ frames are missed. 


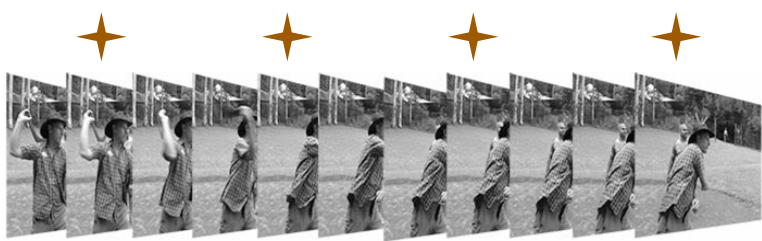

(a)

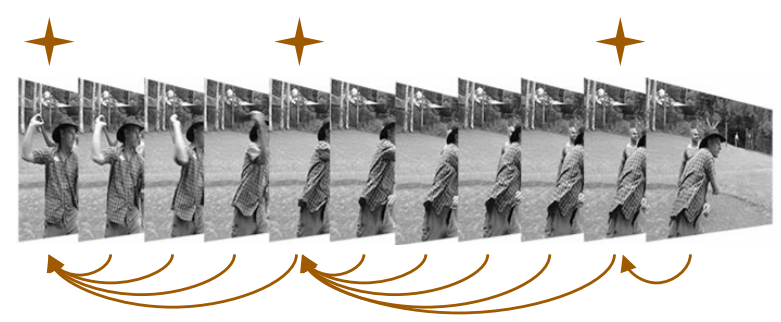

(b)

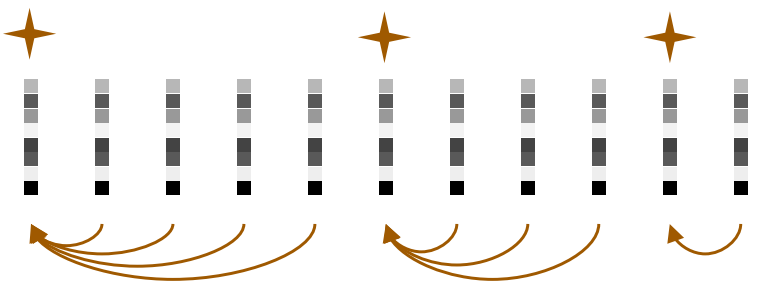

(c)

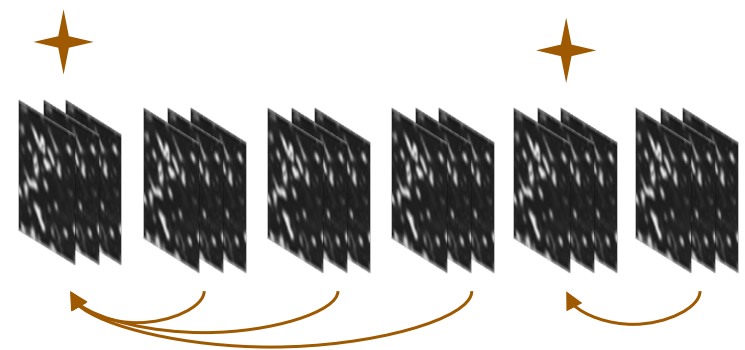

(d)

Fig. 2. Illustration of mask update indicators. The stars denote when masks are updated. The arrows mark how successive frames are compared against the frame of the previous update. 2a: Regular update. 2b: Update when MSE on input images exceeds threshold. 2c: Update when MSE on output activations exceeds threshold. $2 \mathrm{~d}$ : Update when the number of activation sign changes exceeds threshold. See Sec. II-B for details.

2) Input MSE: A more flexible criterion that accounts for direct changes in input is by computing the pixel-wise square difference on input images, i. e. , the Mean Square Error (MSE) between the current frame and the frame at the time of the last update (Fig. 2b). If the MSE value surpasses a given threshold, a mask update is triggered. A potential drawback of this method is that the MSE input criterion may be set off by global changes in lighting etc., which are not necessarily related to changes in the object of interest.

3) Output MSE: Similarly, we can compute the MSE between the network output for the current frame and the output for the frame at the previous update time. If the MSE exceeds a given threshold (which may vary from the MSE input threshold), the masks are updated for the next frame (Fig. 2c). A drawback is that the MSE output criterion triggers updates for the next sample, i. e. with one frame delay.

4) Number of Activation Sign Changes: Activation masks can be applied to all the layers in a network as shown in (3), or to a subset of layers. For instance, one can choose to mask and contract only the upper half of a network. In that case, we can apply a fourth criterion to predict mask updates. Mask updates become necessary when individual neurons in a layer change the sign of their activation. If only the layers from layer $j$ upwards are masked, we can count the number of neurons in layer $j$ that changed the sign of their activations since the time of the last mask update. A mask update is triggered when more than a certain fraction of the neurons changed their activation sign (Fig. 2d). Among the update criteria discussed so far, this predictor correlates most strongly with the actual validity of a mask for a given input sample.

In Section III we evaluate how well each of these four update criteria maintains the accuracy of the network while sweeping the update threshold.

In practice, we determine the threshold by means of a simple calibration phase. For the MSE input for instance, we collect the MSE between the frames of a video sequence from the training set, and plot the histogram of MSE values. The threshold is then chosen as the median (or some percentile) of that MSE distribution. The threshold is applied globally to all masked layers of the network, and stays constant for the duration of the experiment.

\section{Implementation Details}

A brief description of the workflow for compiling and using the contracted network is presented here. Given a network architecture, the network (or network section) is first tested for suitability of contraction. Each layer to be contracted must be transformable into a combination of matrix multiplication, bias addition and mask operations. In particular:

- Fully-connected layers require no modification because their weight matrix is already of rank 2.

- Dropout layers can be removed after training.

- Batch-normalization layers can be integrated into the preceding convolution layer after training.

- Activation layers (inserted after fully-connected or batchnormalization or convolution layers) must be transformable into a mask. For instance, piece-wise linear functions like the leaky ReLU work, but not the exponential linear unit ELU.

- Convolution layers share weights among neurons, so (6) cannot be applied directly. However, the convolution operation can be written in terms of a matrix-vector product by unrolling the convolution using a generalized Toeplitz matrix. In fact, several deep learning libraries (including caffe) utilize an image-to-column transformation to enable convolution via optimized Generalized Matrix-Multiplication (GEMM) methods [31].

- Transposed convolution layers, as used in autoencoders, can be written as convolution layers, where upsampling is achieved by inserting a mesh of zeros in the layer input. 
- Average-pooling layers can be written as convolution layers and then unrolled as such.

- Max-pooling layers can likewise be written as convolution layers, but with a mask prepended, which gates the maximum activation of each pooled patch. This mask is then updated in the same way as the activation masks of ReLU layers.

- Networks with parallel branches, like skip-connections in ResNets or inception modules in GoogLeNet, can be contracted by applying each branch separately on a copy of the input to a multi-branch section.

With the layer transformations above, the masking and contraction method can be applied to a wide range of architectures, as demonstrated in Sec. III. An architecture that cannot be contracted is for instance U-Net [32] due to its high inter-layer connectivity. However, architectures with interlayer connections can often still be used by contracting only a subset of the layers. Such partial contraction is beneficial also to maintain the non-linearity of the network function and reduce the number of mask updates required at runtime.

After the initial checks and layer transformations, the network to be contracted now consists of a set of triplets $\left(\tilde{W}^{k}, \tilde{\mathbf{b}}^{k}, \mathbf{m}^{k}\right)$, one triplet for each layer. The tilde symbols denote that the weights and biases may have been modified through unrolling, absorption of batch-normalization parameters, etc. The masks may be initialized with a random vector or may be directly obtained from the first sample of the sequence. These triplets will be used during inference to compute the contracted $(Q, \mathbf{q})$ parameters. During inference, we iterate through the sequence sample by sample, and compute the network output following (8). For each sample, we also check whether the reference point $\mathbf{x}_{*}$ should be reset using one of the criteria in Sec. II-B. The following pseudo-code summarizes the workflow:

Input: Feed-forward network $F(\mathbf{x})$; sequential dataset $\mathcal{X}$. Output: Contracted network $C_{F}(\mathbf{x})$; network output $\mathcal{Y}$.

Transform each layer into triplet $\left(\tilde{W}^{k}, \tilde{\mathbf{b}}^{k}, \mathbf{m}^{k}\right)$ (Sec. II-A).

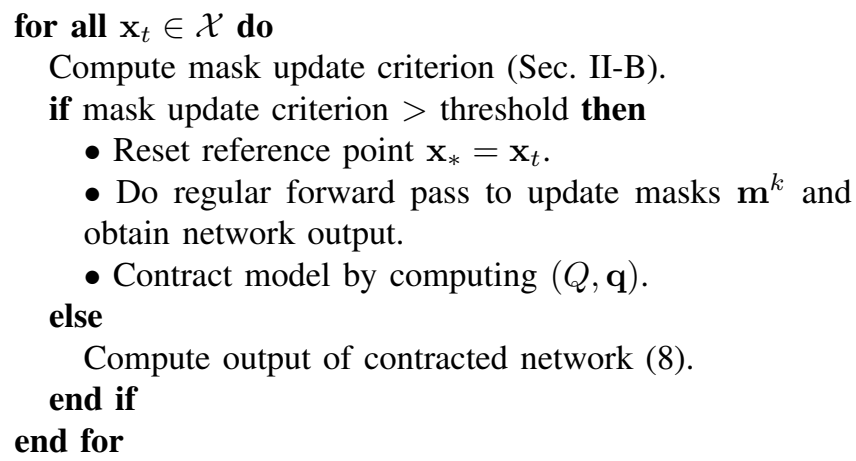

\section{Computational Cost}

The inference cost of a contracted network is compared against that of a standard multi-layer network. In a contracted network, the cost comes from the multiplication of the (flattened) input samples with the $Q$ matrix and addition of the bias $\mathbf{q}$ (and possibly the application of a final activation function on the resulting output values). Assuming a $Q$ matrix of dimension $\left(N_{0} \times N_{n}\right)$ (where $N_{0}$ is the number of neurons in the first masked layer and $N_{n}$ the number of neurons in the final masked layer), and a bias vector $\mathbf{q}$ of $N_{n}$ elements, the number of computations needed for inference is

$$
\mathcal{C}_{\text {contracted }}=2 N_{0} N_{n}+N_{n}=\left(2 N_{0}+1\right) N_{n} .
$$

In contrast, the number of computations for the original network is proportional to the number of neurons in each layer times their fan-in $f_{\text {in }}$ (i.e. the number of incoming connections):

$$
\mathcal{C}_{\text {standard }}=\sum_{k}^{n} 2 N_{k} f_{\mathrm{in}, k}+N_{k}=\sum_{k}^{n}\left(2 f_{\mathrm{in}, k}+1\right) N_{k} .
$$

The inference cost in the contracted model only depends on the sizes of the input and output, whereas the inference cost in a multi-layer ANN scales with the number of layers and its internal connectivity. This fact becomes increasingly important in networks with hundreds of layers such as Inception and ResNet. On the other hand, the proportionality factors $N_{0}$ and $N_{n}$ in (12) may become prohibitively large in tasks like object detection or scene segmentation, where multi-channel heat maps are produced as output instead of the output of a limited number of classification neurons.

Having determined the inference cost of individual samples, we now factor in the mask updates to derive the total inference cost of the contracted network. To obtain the activation pattern needed for the masks, one can simply perform a standard forward pass in the original network. Another forward pass with the contracted network is not necessary for the current sample because the output of the original network can be used directly. The newly obtained masks allow updating of the $(Q, \mathbf{q})$ parameters, which are used for inference with the subsequent samples. In a dedicated hardware implementation, this computation of the contracted parameters can occur in the background, using spare compute cycles, even on the host controller on a separate idle thread. That way, the updates of the masks occur concurrently with the low-latency forward passes for inference and do not impede latency. We therefore consider the cost of inference in the contracted model at the time of an update to be that of a forward pass in the original network, $\mathcal{C}_{\text {standard }}$.

The cost of computing a mask update indicator (like MSE input) in the worst case consists of computing the MSE on two feature maps, which is negligible compared to the inference cost.

Let $r_{\text {update }}=$ \#updates/\#samples denote the mask update rate and

$$
r_{\mathcal{C}}=\frac{\mathcal{C}_{\text {no-update }}}{\mathcal{C}_{\text {update }}}=\frac{\mathcal{C}_{\text {contracted }}}{\mathcal{C}_{\text {standard }}}
$$

the cost ratio of a forward pass without and with mask updates. The compression rate is then given by

$$
r=r_{\mathcal{C}}+r_{\text {update }}\left(1-r_{\mathcal{C}}\right) .
$$

In the limit where the contracted network cost is much smaller than the cost of the original network $\left(r_{\mathcal{C}} \ll 1\right)$, 
the compression rate is simply given by the update rate: $r \approx r_{\text {update. }}$. There are two limit cases in which the network contraction method brings little to no gain, i. e. $r \approx 1$. The first case occurs if the masks need to be updated for almost every sample, i.e. $r_{\text {update }} \approx 1$; the second case if the cost of the contracted network is about the same as the original network, i.e. $r_{\mathcal{C}} \approx 1$.

As a first example, consider LeNet-5 [33], a 4-layer convolutional neural network with 1.2 million parameters, which receives as input gray-scale images of dimension $28 \times 28 \times 1$. The labelled output of the network falls into one of 10 classes. Then, $Q$ is a $(10 \times 784)$ matrix, and $\mathbf{q}$ is a bias vector of size 10 . In one forward pass, $\mathcal{C}_{\text {contracted }}=15.6 \mathrm{kOp}$ and $\mathcal{C}_{\text {standard }}=2340 \mathrm{kOp}$ for each frame.

As second example, the Inception-V3 architecture has an input dimension of $299 \times 299 \times 3$ and 1000 output classes, resulting in an inference cost of $0.53 \mathrm{G}$ and $11.4 \mathrm{G}$ operations for the contracted and original network, respectively.

Assuming that the masks must be recomputed every second frame $\left(1 / r_{\text {update }}=2\right)$, the contracted LeNet-5 and InceptionV3 architectures would see a reduction in computations by $1 / r=2 \times$ and $1.92 \times$, respectively. With the $Q$ matrix updated every 10th frame, the computes are reduced by $1 / r=9.1 \times$ and $7.69 \times$, respectively. Table I lists these compute costs for the tasks discussed in Sec. III.

\section{RESULTS}

We implemented the network contraction method in Python using Keras with the TensorFlow backend. The complete code is available on $\mathrm{GitHub}^{2}$.

We chose a variety of natural video datasets to evaluate the usefulness of this method in different settings, described in the next subsections. Most of the videos contain rich temporal and spatial dynamics, which serves to demonstrate that the mask update mechanism does not miss critical changes but makes optimal use of static episodes. In some datasets, ground-truth is available so we can explicitly measure how the model accuracy is affected by the masking and contraction. Where no ground truth is present, we use the output of the original network as reference for the masked model. Example videos from our results are available online to illustrate the performance of the contracted networks, compare them to the original networks and, where applicable, to the ground-truth. The summarized results in Table I will be discussed with each subsection describing a particular dataset.

\section{A. Road Detection}

Dataset: As first task, we consider road detection on KITTI driving data [2]. The KITTI benchmark suite contains a dedicated dataset for road detection based on images. Since our method targets video, we use the KITTI multi-object tracking dataset, which consists of 29 videos of urban driving scenes. For ground truth, we use the road segmentation masks obtained

${ }^{2}$ Link will be published on acceptance of the paper.

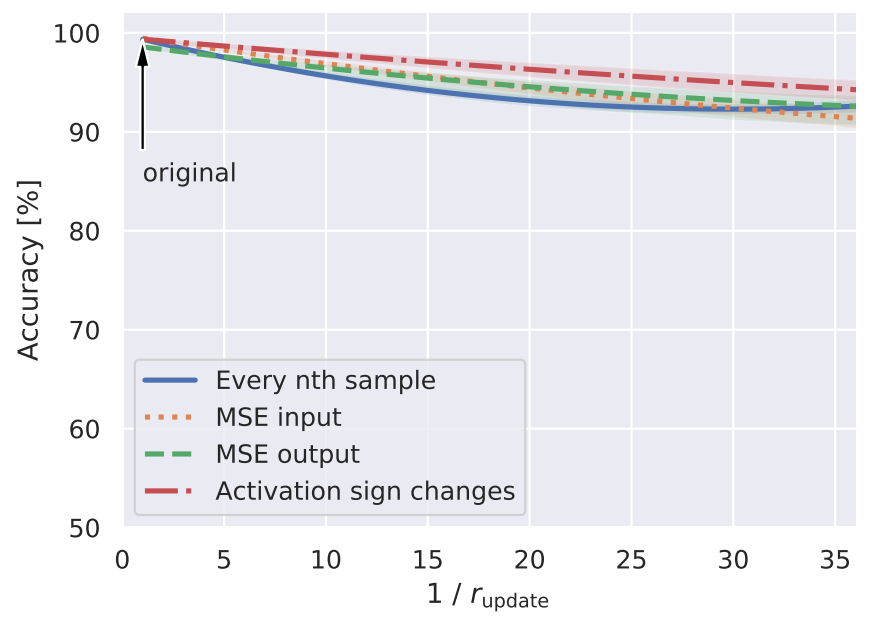

Fig. 3. Pixel-wise accuracy of the contracted network relative to the original model, as a function of the mask update rate, for the road detection task.

by running the original model before contraction. The pixelwise accuracy reported in Table I is relative to the accuracy from the original model.

Model: The architecture used in this task is a Fully Convolutional Network (FCN) [34], which achieved state-of-the-art performance on common semantic segmentation benchmarks. The model consists of a VGG-16 base, whose fully-connected layers are transformed into convolutional layers to produce a low-resolution classification heatmap over the input image. This heatmap is then upsampled to the original input resolution via a deconvolution layer, resulting in a segmentation mask.

Contraction: The FCN architecture is well suited for the proposed contraction method because it contains a lowdimensional bottleneck: The final encoder layer before the deconvolution. Such a bottleneck keeps the size of the contracted $Q$ matrix small (c.f. Eq. 6). We contract the network starting from the third convolution layer up to the decoder, thereby replacing 14 convolution layers with a single fully-connected layer. The number of weights within this contracted layer is only $1 \%$ smaller than those of the original 14 convolution layers, but the number of neurons is reduced from $8.5 \times 10^{6}$ to 180 , and the number of operations needed during inference is reduced from $61 \times 10^{9}$ to $265 \times 10^{6}$ in the contracted part.

Mask updates: To evaluate how the accuracy of the contracted model on the road detection task depends on the mask update rate, we varied the threshold hyperparameter for the four update indicators and measured the relative pixelwise accuracy of the contracted model to that of the original model (Fig. 3). The contracted model maintains high relative accuracy $(>95 \%)$ with only one mask update for every 35 frames on average. The four update indicators perform similarly. Using regular mask updates for every $n$th sample gives the worst results. Using mask updates based on the number of activation sign changes gives the highest accuracy, which is expected because of this metric's direct correlation with the activation masks. Fig. 4 illustrates the road segmentation results from the original and contracted models. 
TABLE I

SUMMARY OF EXPERIMENTS

\begin{tabular}{|c|c|c|c|c|c|c|}
\hline $\begin{array}{l}\text { Dataset } \\
\text { Task }\end{array}$ & & $\begin{array}{l}\text { KITTI } \\
\text { Road detection }\end{array}$ & $\begin{array}{l}\text { CAVIAR surveillance } \\
\text { Pose estimation }\end{array}$ & $\begin{array}{l}\text { KITTI } \\
\text { Object detection }\end{array}$ & $\begin{array}{l}\text { ImageNet video } \\
\text { Object detection }\end{array}$ & $\begin{array}{l}\text { t-MNIST } \\
\text { Denoising }\end{array}$ \\
\hline Architecture & $\begin{array}{l}\text { original } \\
\text { masked }\end{array}$ & $\begin{array}{l}17 \text { Conv } \\
3 \text { Conv, } 1 \text { FC }\end{array}$ & $\begin{array}{l}90 \text { Conv } \\
10 \text { Conv, } 2 \text { FC }\end{array}$ & $\begin{array}{l}23 \text { Conv } \\
16 \text { Conv, } 1 \text { FC }\end{array}$ & $\begin{array}{l}23 \text { Conv } \\
16 \text { Conv, } 1 \text { FC }\end{array}$ & $\begin{array}{l}5 \text { Conv, } 2 \text { FC } \\
2 \text { Conv, } 2 \text { FC }\end{array}$ \\
\hline Dimensions & $\begin{array}{l}\text { input } \\
\text { output }\end{array}$ & $\begin{array}{l}80 \times 288 \times 64 \\
5 \times 18 \times 2\end{array}$ & $\begin{array}{l}36 \times 48 \times 128 \\
36 \times 48 \times 57\end{array}$ & $\begin{array}{l}13 \times 13 \times 512 \\
13 \times 13 \times 1024\end{array}$ & $\begin{array}{l}13 \times 13 \times 512 \\
13 \times 13 \times 1024\end{array}$ & $\begin{array}{l}16 \\
28 \times 28 \times 1\end{array}$ \\
\hline Metric & $\begin{array}{l}\text { original } \\
\text { masked }\end{array}$ & $95 \%$ acc. rel. to orig. & 2.88e-4 MSE to orig. & $91.55 \mathrm{mAP}$ rel. to orig. & $\begin{array}{l}91.51 \mathrm{mAP} \\
88.75 \mathrm{mAP}\end{array}$ & $\begin{array}{l}0.016 \mathrm{MSE} \\
0.023 \mathrm{MSE}\end{array}$ \\
\hline Comput. reduction & $1 / r_{\mathcal{C}}$ & $3.9 \times$ & $2.1 \times$ & $1 \times$ & $1 \times$ & $22.7 \times$ \\
\hline Mask updates & $1 / r_{\text {update }}$ & 35 & 10.1 & 2 & 40 & 3 \\
\hline Compression & $1 / r$ & $3.6 \times$ & $1.9 \times$ & $1 \times$ & $1 \times$ & $2.8 \times$ \\
\hline
\end{tabular}

\section{Original}

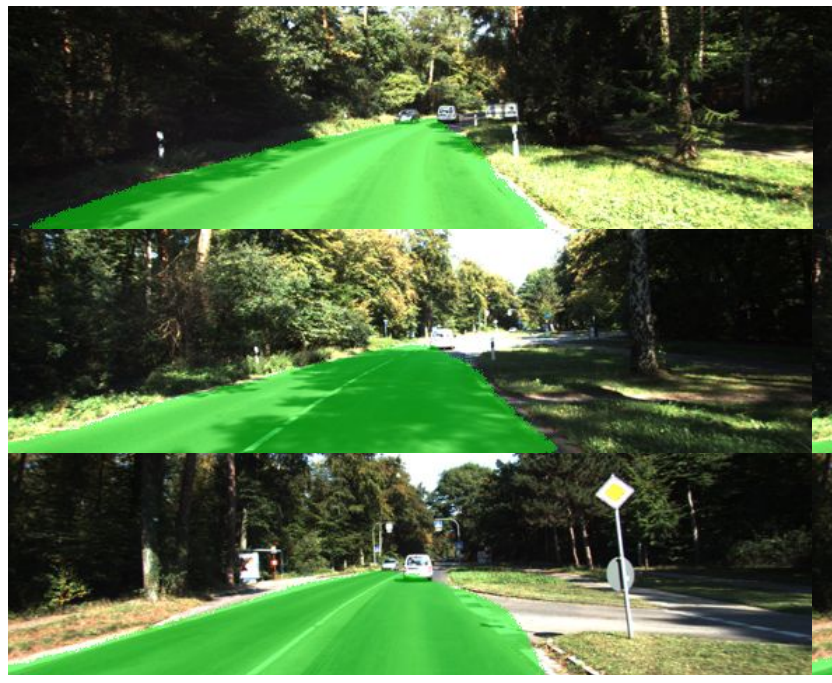

Contracted

Mask update every 39 frames on average (MSE input)

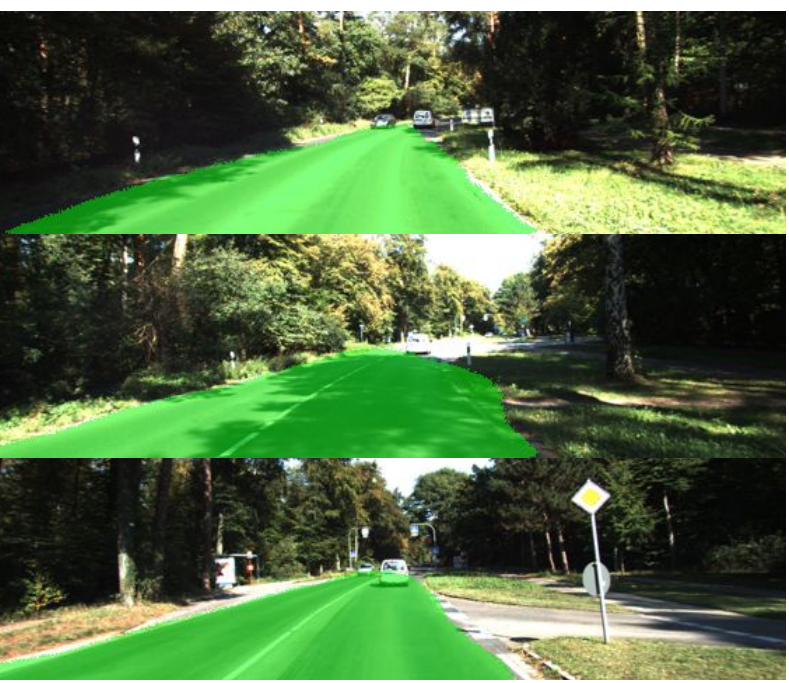

Fig. 4. Examples of road segmentation masks (in green) from the original (left column) and the contracted (right column) models in the road detection task. (Best viewed in color.)

\section{B. Pose Estimation on Surveillance Data}

Dataset: The videos in the CAVIAR surveillance dataset ${ }^{3}$ were recorded with a wide angle lens along the hallway of a shopping center in Lisbon. The video frames have a resolution of $384 \times 288$ pixels and are recorded at 25 frames per second (fps). We use the same seven test videos as related work [7].

Model: We use the OpenPose network for a pose estimation task [35]. The architecture consists of a VGG-like trunk of 12 convolution layers and 3 pooling layers. The network then splits up into two branches, one for identifying body parts, the other for connecting them appropriately. Both branches consist of 5 convolution layers followed by 5 stages of 7 convolution layers each, for a total of 40 convolution layers per branch.

Contraction: We apply feature masking on both branches, but not on the trunk, for reasons that will become apparent in the next paragraph. The resulting architecture consists of the trunk plus two fully-connected output layers replacing

${ }^{3}$ CAVIAR was funded by the EC project IST 200137540 and is available online (http://homepages.inf.ed.ac.uk/rbf/CAVIAR/) the two 40-layer branches. The two branches of the original network require 155 billion operations in total, which are reduced to 44 billion operations after contraction. Together with the 61 billion operations for the trunk, the total reduction in computations is $2.06 \times$.

Mask updates: To quantify which layers are likely to require few mask updates, we count the number of neurons for each layer that switch activation sign between consecutive frames, and average this number across all video sequences of the test set. The result is shown in Fig. 5. We observe a trend as described in [36], where contiguity (the sequence length of activations with the same sign) increases with network depth. In other words, neurons in higher layers (past the 12th layer) tend to maintain the same sign from frame to frame. This observation is in line with the common observation that higher layers which encode more high-level concepts are expected to be stable across longer time periods. Further support was offered recently by [37], who applied the firstorder Taylor expansion to each residual block of a ResNet architecture and showed that while lower layers learn new 


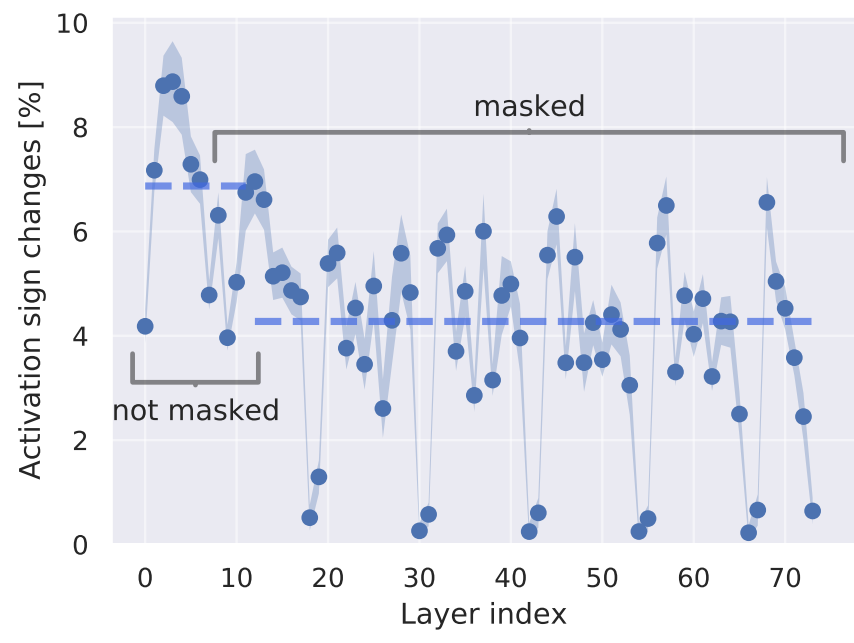

Fig. 5. Percentage of neurons that change their activation sign value between consecutive frames. Numbers are averaged over test sequences of the pose estimation task. The shaded region denotes the 3 times the standard deviation. The first 12 convolution layers are not masked, the remaining 68 are masked. Dashed lines represent the median number of activation sign changes for both the masked and non-masked layers. The median value for masked layers is $40 \%$ lower than that of the masked layers. The low peaks correspond to the $1 \times 1$ convolution layers.

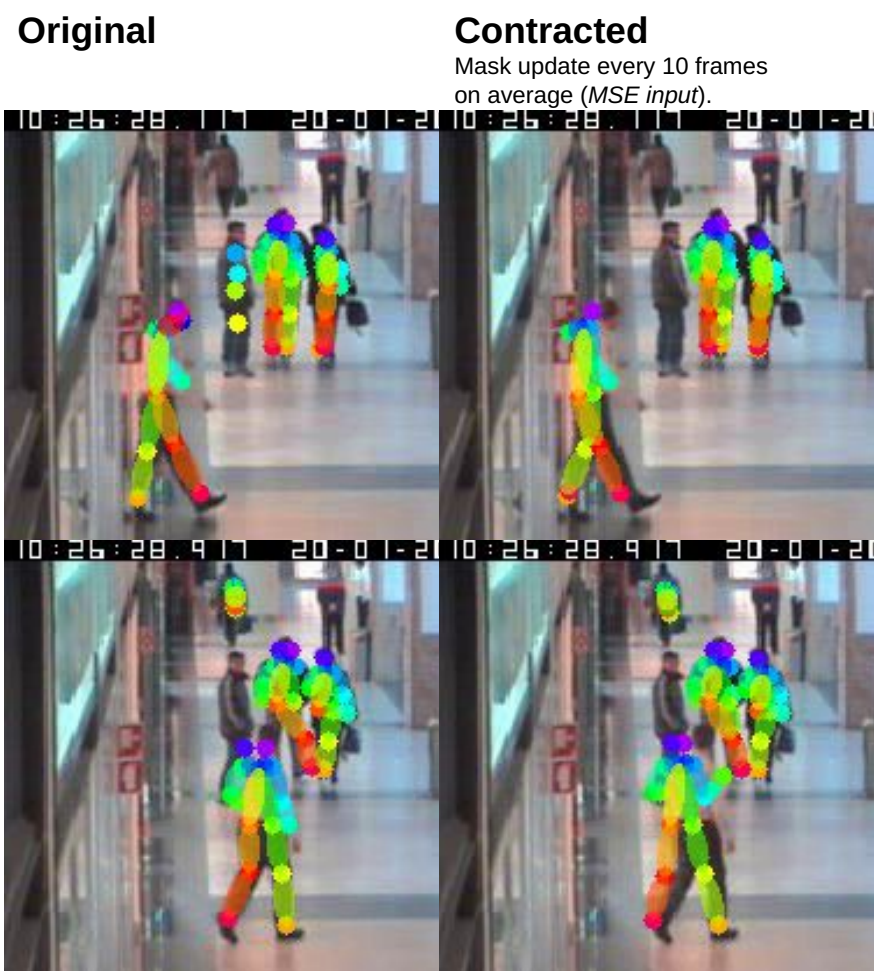

Fig. 6. Example comparison of the pose estimation of the original and the contracted model. (Best viewed in color.)

representations, higher layers tend to iteratively refine the hierarchical representations of lower layers. This, together with the $40 \%$ fewer activation sign changes in the upper two branches of the present architecture, motivates our choice to contract these layers but not the VGG trunk.

It is advantageous for the network contraction when the

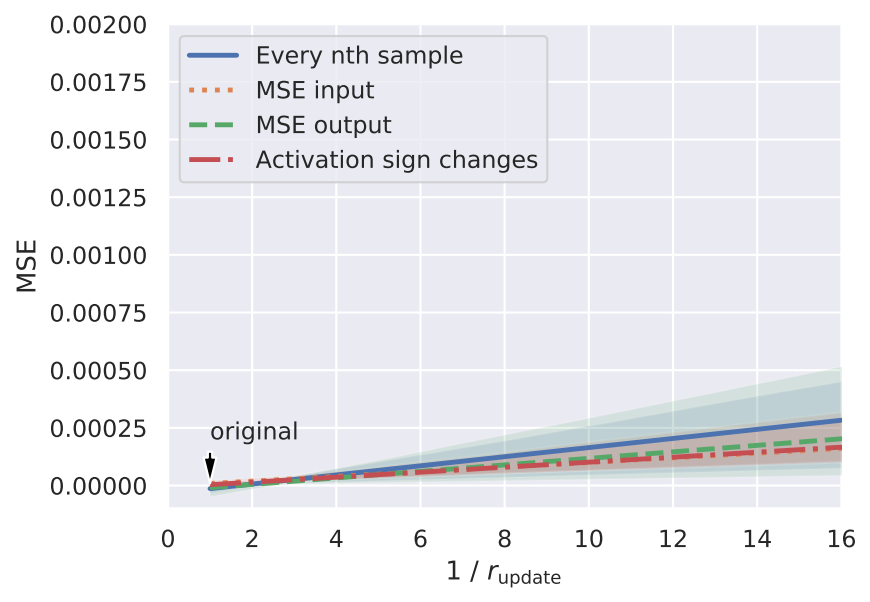

Fig. 7. Mean square error between the contracted and original network as a function of the mask update rate, $r_{\text {update }}$, for the pose estimation task. The shaded region denotes the 3 -sigma confidence interval of the regression line for the 7 test sequences.

activation sign changes are correlated between layers, because this allows the mask update step to be done synchronously across all layers. For each video sequence in the test set, we compute the correlation between activation sign changes in the first layer versus all other layers and obtain a median correlation value of 0.95 . This result indicates that a mask update trigger is appropriate for updating all masks simultaneously.

To quantify the performance of the contracted network, we measured the MSE between the output of the masked network and the original OpenPose network, while gradually increasing the threshold for mask updates, thereby trading off accuracy vs computational cost. Fig. 7 depicts this trade-off, and compares the performance of each of the four proposed update indicators. Overall, the MSE increases only moderately with decreasing update rates, with little dependence on the update mechanism. Updates based on dynamic indicators lead to slightly increased performance compared to updates at regular intervals. The improved behavior of the MSE input metric is not surprising: With static indoor surveillance videos, MSE input is less likely to be distracted by task-unrelated changes in the scene (see Fig. 6 for an illustration).

For the two sequences that gave the best and the worst results, we provide online videos that show the pose estimation results at different update rates ${ }^{4}$. The reduction in computations and the relative increase in error are listed in Table I for the lowest performing sequence.

In future work, our method could include a preprocessing strategy as proposed by [38]. In this work, the authors train a generic CNN on a stack of video frames to track the $2 \mathrm{D}$ human upper body pose on a dataset of sign language gestures. They report improved accuracy when subtracting the per-video mean from input images, thereby removing video-specific static background and improving generalization. 


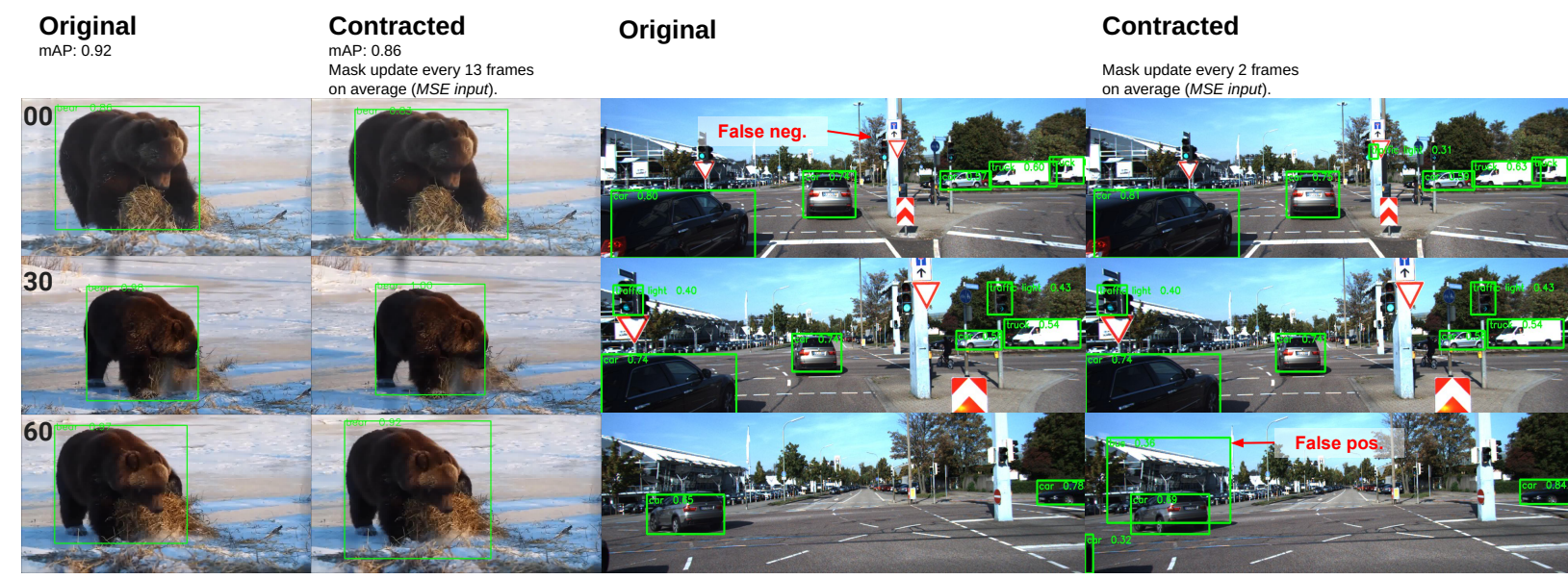

Fig. 8. Illustrative comparison of the original and the contracted model output for the object detection task using ImageNet (left) and KITTI (right).

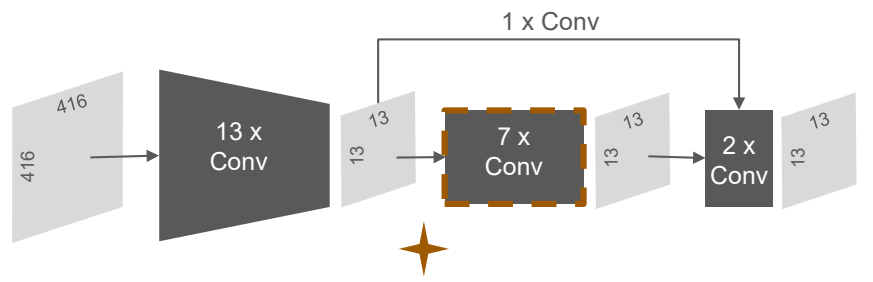

Fig. 9. YOLO architecture used for object detection in the KITTI and ImageNet video experiments. The upper seven convolution layers (dashed border) are masked. Sign changes in activations of the first masked layer predict mask updates (indicated by star). The light gray boxes denote the feature map size of the final layer of a convolution block.

\section{Video Object Detection}

Datasets: The two datasets are the KITTI dataset and ImageNet object-detection-from-video challenge. The object tracking test set of the KITTI Vision Benchmark Suite [2] consists of 29 videos of urban driving scenes; typical objects to be tracked include cars, buses, pedestrians, bicycles, traffic lights and street signs. The labels provided for the ImageNet dataset do not all match the classes of the $\mathrm{COCO}$ dataset with which our model was trained, so we selected a subset of ImageNet videos where the labels matched the known classes. Fig. 8 illustrates the masking method applied on two randomly selected clips from the two datasets.

Model: We use the YOLO architecture [3] pretrained on the COCO dataset ${ }^{5}$. The network shown in Fig. 9, consists of a trunk of 12 convolution layers (interleaved with batch normalization and pooling). The graph then splits into a branch with 7 convolution layers and a skip-connection branch with a single convolution layer. The two branches merge to pass through two more convolution layers, the second of which contains separate channels for the object bounding boxes, the confidence scores, and the 80 different class predictions.

Contraction: The feature masking method is applied to the upper block of 7 convolution layers (dashed box in Fig. 9). A

\footnotetext{
${ }^{4}$ https://www.youtube.com/playlist?list=PLUK3dDFZv51JlzP_ SH41h7Fws-aefyuCK

${ }^{5}$ http://cocodataset.org
}
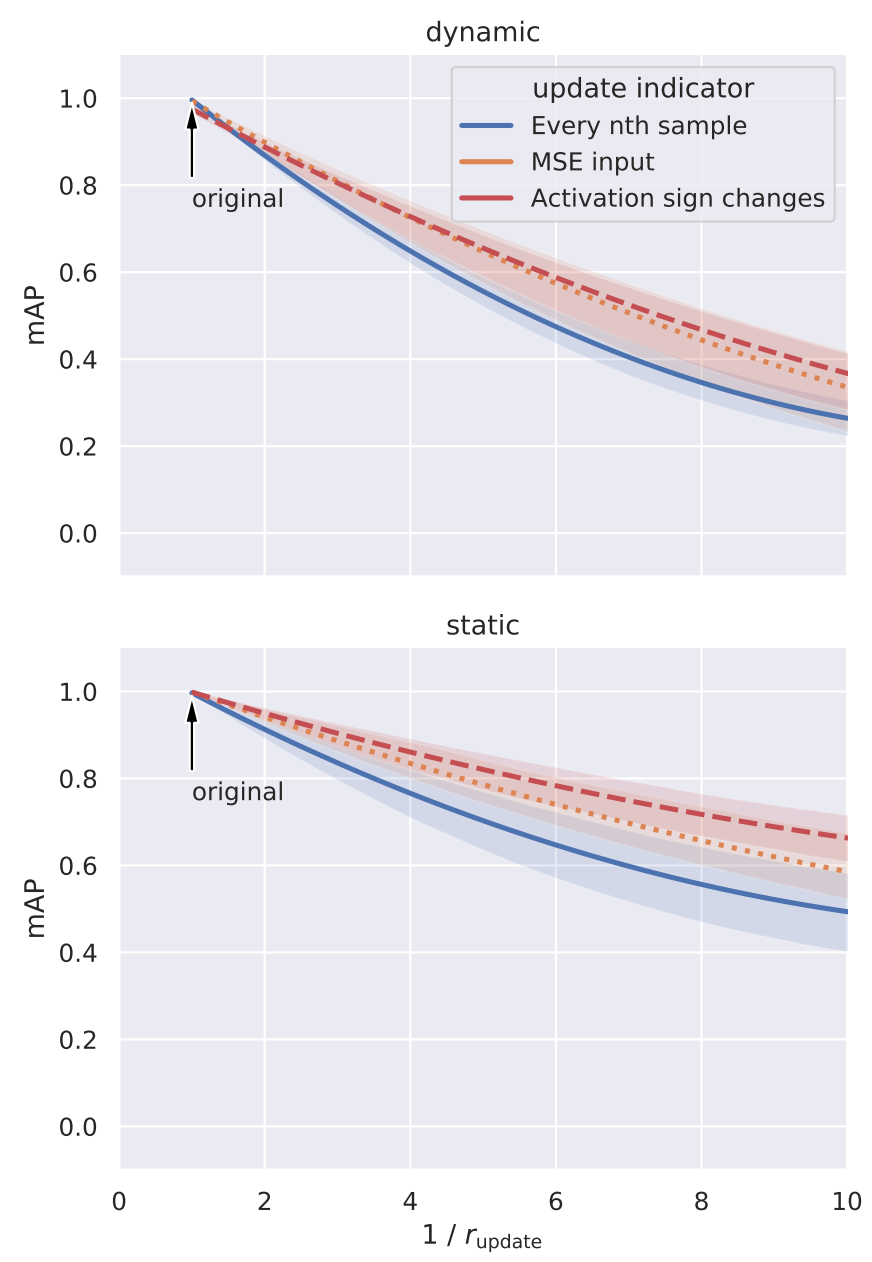

Fig. 10. mean Average Precision (mAP) plotted against the mask update rate for the KITTI object detection task. Each line is obtained from a linear regression of the mAP values across the test samples. The shaded region denotes the 3-sigma confidence interval. We used the labels and bounding boxes predicted by the original model as ground truth for the contracted model. The two panels separately show videos taken from a moving car $(20$ clips, top) and stationary car ( 9 clips, bottom). 


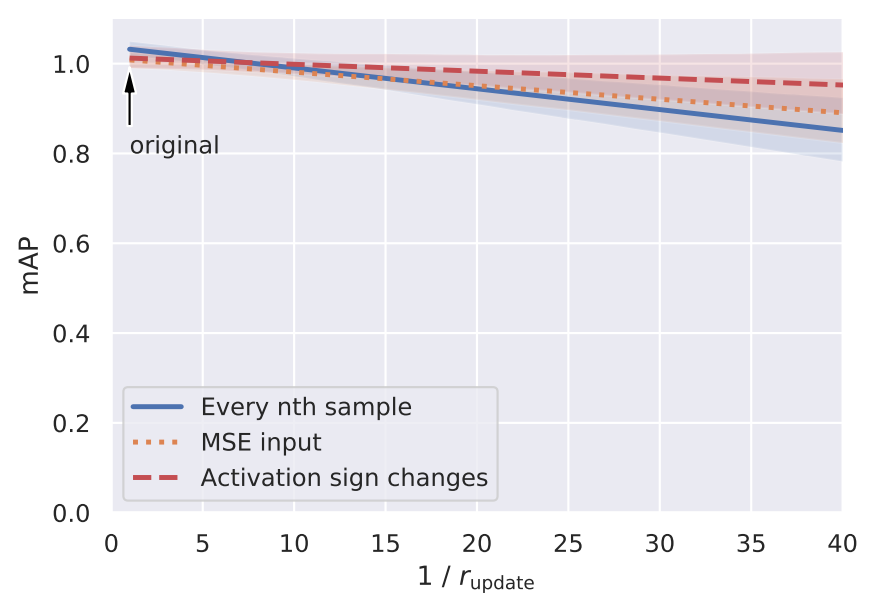

Fig. 11. Least-squares fit of the mean Average Precision for the ImageNet video task. The reported mAP values of the contracted model were normalized by the mAP of the original model to facilitate the regression.

challenge in applying the network contraction method to this architecture is that both the input and the output dimensions of the masked section are large (c.f. Fig. 9), resulting in a large $Q$ matrix (6 billion entries). The size of the $Q$ matrix is the reason why the contraction method does not appear to bring a computational advantage over the original model: The matrix-vector product of the input with the $Q$ matrix during inference costs just as much as the forward pass through the 7 uncontracted convolution layers, namely 12 billion operations. Albeit the YOLO architecture may not be suited for our network contraction approach, this experiment is nevertheless worthwhile for showing that our dynamic feature masking can be applied successfully to the task of multiple object tracking.

Mask updates: As before, we analyze the performance tradeoff by measuring the mean Average Precision (mAP) while varying the update rate, using our proposed update indicators. For the MSE input metric we take the input to the contracted block of the network. For the number of activation sign changes, we use the activations of the first layer of this block as indicated by a star in Fig. 9. The MSE output criterion was not applicable here because of the multi-functional structure of the output layer.

The contracted model is more robust to low update rates in static scenes (recorded from a stationary car, Fig. 10 bottom) than in dynamic scenes (taken from a moving car, Fig. 10 top). In both situations, updates triggered by activation sign changes achieve highest mAP, regular update rates achieve lowest.

Annotated videos of both the original and our masked model are provided for visual comparison ${ }^{6}$.

For the ImageNet video dataset, the performance trade-off (Fig. 11) is qualitatively similar to the KITTI task, with the update metrics ranking in the same order. The similar performance of the MSE input and activation sign changes metric is supported by the observation that the Pearson correlation coefficients between the two quantities lie above 0.9 for each

\footnotetext{
${ }^{6}$ https://www.youtube.com/playlist?list=PLUK3dDFZv51KGIT5h1iJLp_ JxtJ7BKn-
}

of the masked layers. On average, the mAP remains within $10 \%$ of the precision of the original model for update rates beyond 40 frames.

\section{Denoising Autoencoder}

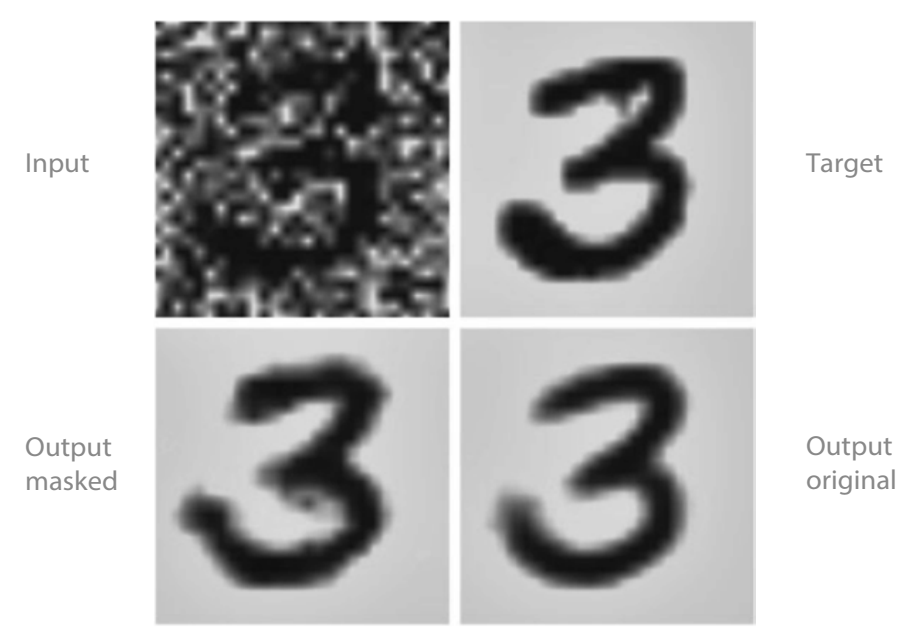

Fig. 12. Illustration of the denoising autoencoder task, with the noisy model input (top left), training target (top right), denoised output of the original model (bottom right), and output of the contracted model (bottom left).

Dataset: The temporal MNIST (t-MNIST) dataset is a version of the MNIST handwritten-digit dataset [33] ordered by similarity.

Model: The task is to denoise images from this dataset with added Gaussian noise (Fig. 12). The network is a Denoising AutoEncoder (DAE) where the encoder consists of two convolution layers followed by a fully-connected layer, mapping the $28 \times 28$ gray-scale input images into a 16-dimensional latent space. The decoder section consists of a fully-connected layer and three transpose-convolution layers, which map the 16-dimensional encoded representation back into image space.

Contraction: Only the decoder is contracted to benefit from the low-dimensional latent representation. As in the other experiments (see e.g. Fig. 5), the number of activation sign changes tends to decrease from lower to higher layers, which further motivates masking upper parts of the network. The resulting architecture consist of two convolution layers and two fully-connected layers, the last being the contracted decoder.

In general, autoencoders are advantageous for network contraction because the latent representation of the DAE architecture is low-dimensional, resulting in $2.2 \times$ fewer parameters, $4.1 \times$ fewer neurons, and $22.7 \times$ fewer operations for this architecture (c.f. Table I), while maintaining good denoising performance ${ }^{7}$.

Mask updates: The update indicator based on number of activation sign changes could not be used in this experiment because the latent representation (which feeds into the contracted part) does not use a ReLU function. The MSE input metric appears to be a good predictor of when to update the masks, as indicated by a high correlation between the MSE

\footnotetext{
${ }^{7}$ https://youtu.be/bBFTkyckQe8
} 


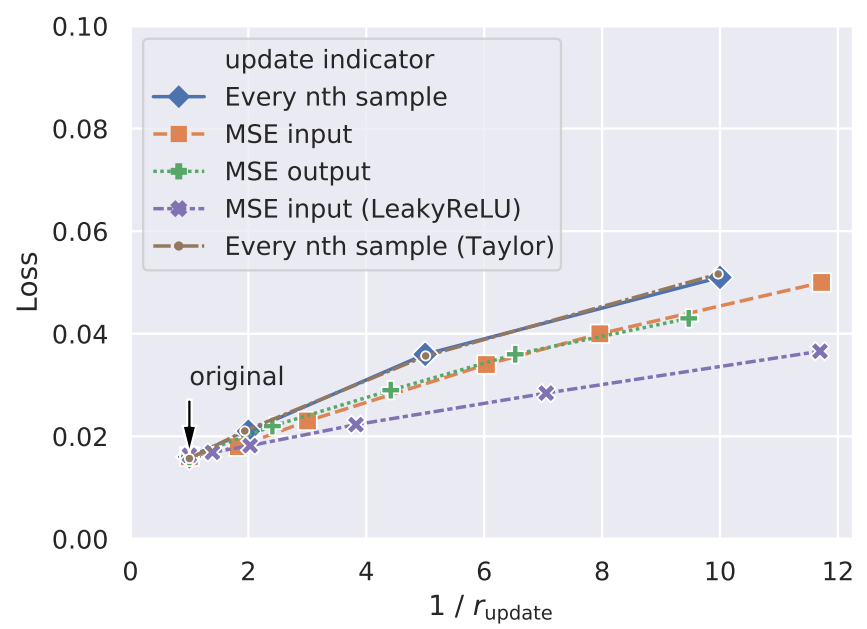

Fig. 13. Loss (reconstruction MSE) plotted against the mask update rate for the DAE task on t-MNIST.

input and the number of activation sign changes in the two transposed convolution layers of the decoder. The dynamic criteria (MSE input / output in Fig. 13) perform consistently better than a fixed update at every $n$-th frame, which is in line with previous observations (see e.g. Figures 7, 10, and 11).

Taylor approximation: The first-order Taylor expansion of the original network behaves just as the contracted model (as indicated by the overlapping blue and brown upper lines in Fig. 13), which supports the relation of our method with Taylor approximations derived in Sec. II-A.

\section{E. Retraining with ReLU Variant}

The method of contracting several layers of a network into a single $Q$ matrix using dynamically updating activation masks can be applied to a pre-trained feed-forward network without retraining. However, one may consider retraining the network to reduce the number of mask updates needed during inference.

We consider the use of leaky ReLU activation functions. An inaccurate mask introduces errors during inference by blocking some positive activations or transmitting some negative activations. These errors are most notable if the masks are used to replace non-leaky ReLUs. For leaky ReLUs, the error due to an outdated mask entry gets smaller, the higher the leak $\alpha$. This fact can be used during training, by gradually increasing the slope of the left branch of the ReLU. This process progressively linearizes the network and is thus strictly limited by a leak $\alpha<1$. As $\alpha$ approaches 1 , the network becomes insensitive to errors in the masks, but also loses expressive power. On the other hand, linear networks do not suffer from the "shattered gradients" problem, which is why [36] initialize their networks to "look linear" at first, with the symmetry in the activation function gradually breaking during training.

To explore the effect of leaky ReLUs on the masking, we train a DAE on the t-MNIST dataset while increasing the leak parameter $\alpha$ at certain epochs, which are evident by the kinks in the training curve (Fig. 14). The model classification performance degrades only little when increasing the linearity

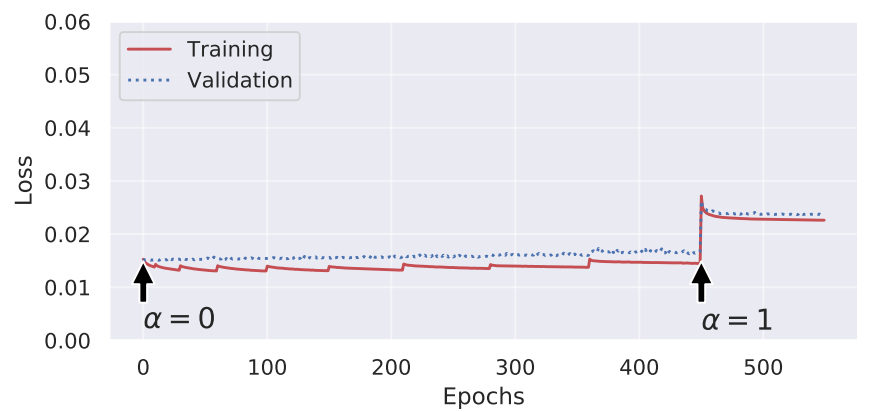

Fig. 14. Accuracy during refinement of the pre-trained DAE on the t-MNIST. During training, the slope parameter $\alpha$ of the Leaky ReLU activation function is increased from $\alpha=0$ at epoch 0 to $\alpha=1$ at epoch 520 .

of the rectifier. A similar convergence behavior is observed when training a 6-layer CNN and a 32-layer ResNet on CIFAR10 (not shown).

After training the decoder of the DAE with increasing linearity, we test our model contraction method on the almost linear model $(\alpha=0.89)$. The result shown in Fig. 13 confirms our initial hypothesis: The contracted leaky ReLU model shows a consistently lower error compared to the contracted ReLU model. The almost linear rectifiers make the model robust to outdated masks.

\section{F. Runtime Measurements}

To estimate the runtime speedup of the contracted model, we measured the execution time of the DAE on a Jetson Nano, an $17-4790 \mathrm{~K}$ CPU, as well as a GeForce GTX $980 \mathrm{Ti}$ GPU. Compared to the original model, the contracted DAE ran on average $2.14 \times$ faster on the Jetson, $2.48 \times$ faster on the CPU, and $2.46 \times$ faster on the GPU. This time does not include the computation of the contracted parameters $(Q, \mathbf{q})$ (Eq. 6), which are recomputed during mask updates (e.g. every third frame for t-MNIST). The contraction step consists mainly of matrix-vector products and is currently implemented in nonoptimized Python code. A preliminary GPU implementation shows that contracting the DAE is $10.1 \times$ faster than computing the forward pass of the original model and is thus not a limiting factor in the total execution time.

\section{Discussion}

The results from the experiments on the different datasets show that the contracted networks maintain good accuracy which is corroborated in the case of the t-MNIST, KITTI, and ImageNet videos where ground truth is available. This finding demonstrates that deep neural networks can be approximated as a first-order Taylor expansion with respect to the input around a dynamically updating reference point (c. f. Sec. II-A).

Even though we demonstrate the applicability of the proposed method on a wide range of tasks and architectures, there are limitations in using the network contraction algorithm. First, the method builds on spatio-temporal correlations between frames, thus works best on video sequences with a high degree of redundancy, such as surveillance and highway driving. Second, for some networks, only a part of the model can 
be contracted, for instance in the presence of skip-connections (c. f. Sec. II-C for a list of supported network features). Third, when a stack of convolution layers are contracted, they are unrolled (giving up weight sharing) and afterwards replaced by a combined fully-connected layer. As a consequence, when the size of both the input and output of the contracted network section is large, the size of the contracted weight matrix may also be large. We quantified this cost in Sec. II-D and evaluated in Sec. III examples of architectures that are less suited (YOLO) and well suited for contraction (autoencoders, FCNs). Even though there are newer architectures that now give better accuracy on the segmentation task (e.g. [39]), the simpler form of the FCN is more suited to edge applications with constrained computing resources [40], which we target with our approach.

We found that the contracted networks lead to a compression factor of $1-3.6 \times$ depending on their architecture. The contracted YOLO network maintains high accuracy over a wide range of mask update rates (on average every 40th frame) for the ImageNet object detection task. However, the large input and output sizes of the YOLO architecture result in a large contracted network, which cannot be supported by available memory in embedded systems (c.f. paragraph Contraction in Sec. III-C). The best architectures for contraction have either a low-dimensional input or output, or an intermediate bottleneck layer, as in the fully-convolutional network for KITTI street segmentation, or the autoencoder for denoising tMNIST. Contractive autoencoders [41] offer additional benefits because their loss function contains a term proportional to the Frobenius norm of the encoder's Jacobian. This penalizer encourages the mapping to the latent space to be contractive in the neighborhood of the training data, making the encoded representation robust to small changes of the input. We hypothesize that a contracted decoder that receives these stabilized latent representations as input will have to update its masks less frequently.

Mask updates can be performed at regular intervals or triggered dynamically based on a specific indicator metric. The indicator based on the number of activation sign changes is most closely correlated with outdated masks, and cheaper to compute than using the MSE input / output metrics. This indicator also requires the least mask updates to maintain model accuracy on the pose-estimation, road detection, and object detection tasks on KITTI and the ImageNet video datasets. This indicator was not applicable in the denoising task because the indicator layer has no ReLU. An alternative is the data-driven predictor MSE input, which achieved better model accuracy than regular updates at every n-th frame.

\section{CONCLUSION}

We present a network approximation scheme that replaces the layer nonlinearities by dynamically updated masks, and show its equivalence to a first-order Taylor expansion through theory and experiments. Among the four mask indicators, our results show that the optimal indicator is based on the count of activation sign changes, is cheap to compute and requires fewer updates at iso-accuracy than the other indicators. By compressing a network using this masking technique, the computational cost of processing a stack of layers is reduced to the cost of performing a single matrix-vector multiplication.

\section{ACKNOWLEDGMENT}

This work has been supported by the Samsung Advanced Institute of Technology, the ETH Zurich and the University of Zurich.

\section{REFERENCES}

[1] B. Rueckauer and S. C. Liu, "Linear Approximation of Deep Neural Networks for Efficient Inference on Video Data," in European Signal Processing Conference, 2019.

[2] A. Geiger, P. Lenz, and R. Urtasun, "Are We Ready for Autonomous Driving? The KITTI Vision Benchmark Suite," in Conference on Computer Vision and Pattern Recognition (CVPR), 2012.

[3] J. Redmon, S. Divvala, R. Girshick, and A. Farhadi, "You Only Look Once: Unified, Real-Time Object Detection," Cvpr 2016, pp. 779-788, 2016.

[4] O. Russakovsky, J. Deng, H. Su, J. Krause, S. Satheesh, S. Ma, Z. Huang, A. Karpathy, A. Khosla, M. Bernstein, A. C. Berg, and L. Fei-Fei, "ImageNet Large Scale Visual Recognition Challenge," International Journal of Computer Vision, vol. 115, no. 3, pp. 211-252, 2015. [Online]. Available: http://dx.doi.org/10.1007/s11263-015-0816-y

[5] V. Campos, B. Jou, X. Giro-i Nieto, J. Torres, and S.-F. Chang, "Skip RNN: Learning to Skip State Updates in Recurrent Neural Networks,' in International Conference on Learning Representations, 2018, pp. 1-17. [Online]. Available: http://arxiv.org/abs/1708.06834

[6] D. Neil, J. H. Lee, T. Delbruck, and S.-C. Liu, "Delta Networks for Optimized Recurrent Network Computation," in PMLR, 2017. [Online]. Available: http://arxiv.org/abs/1612.05571

[7] L. Cavigelli and L. Benini, "CBinfer: Exploiting Frame-to-Frame Locality for Faster Convolutional Network Inference on Video Streams," IEEE Transactions on Circuits and Systems for Video Technology, vol. 30, no. 5, pp. 1451-1465, 52020.

[8] A. Mishra, E. Nurvitadhi, J. J. Cook, and D. Marr, "WRPN : Wide Reduced-Precision Networks," in International Conference on Learning Representations, 2018, pp. 1-11.

[9] D. Miyashita, E. H. Lee, and B. Murmann, "Convolutional Neural Networks using Logarithmic Data Representation," arXiv, 2016. [Online]. Available: http://arxiv.org/abs/1603.01025

[10] I. Hubara, M. Courbariaux, D. Soudry, R. El-Yaniv, and Y. Bengio, "Quantized Neural Networks: Training Neural Networks with Low Precision Weights and Activations," arXiv:1602.02830, 2016. [Online]. Available: http://arxiv.org/abs/1602.02830

[11] S. Han, H. Mao, and W. J. Dally, "Deep Compression: Compressing Deep Neural Networks with Pruning, Trained Quantization and Huffman Coding," in ICLR, 2015, pp. 1-13. [Online]. Available: http://arxiv.org/abs/1510.00149

[12] E. Bengio, P.-L. Bacon, J. Pineau, and D. Precup, "Conditional Computation in Neural Networks for Faster Models," in International Conference on Learning Representations, 2016, pp. 1-9. [Online]. Available: http://arxiv.org/abs/1511.06297

[13] J. Lin, Y. Rao, J. Lu, and J. Zhou, "Runtime Neural Pruning," in Advances in Neural Information Processing Systems, 2017, pp. 2178-2188. [Online]. Available: https://papers.nips.cc/paper/ 6813-runtime-neural-pruning

[14] F. N. Iandola, S. Han, M. W. Moskewicz, K. Ashraf, W. J. Dally, and K. Keutzer, "SqueezeNet: AlexNet-level Accuracy with 50x Fewer Parameters and $\backslash$ i0.5MB Model Size," arXiv, pp. 1-5, 2016. [Online]. Available: http://arxiv.org/abs/1602.07360

[15] A. G. Howard, M. Zhu, B. Chen, D. Kalenichenko, W. Wang, T. Weyand, M. Andreetto, and H. Adam, "MobileNets: Efficient Convolutional Neural Networks for Mobile Vision Applications," arXiv:1704.04861, 2017. [Online]. Available: http://arxiv.org/abs/1704.04861

[16] M. McGill and P. Perona, "Deciding How to Decide: Dynamic Routing in Artificial Neural Networks," in International Conference on Machine Learning, Sydney, Australia, 3 2017. [Online]. Available: http://arxiv.org/abs/1703.06217

[17] V. Sze, Y. H. Chen, T. J. Yang, and J. S. Emer, "Efficient Processing of Deep Neural Networks: A Tutorial and Survey," Proceedings of the IEEE, vol. 105, no. 12, pp. 2295-2329, 2017. 
[18] X. Zhu, Y. Xiong, J. Dai, L. Yuan, and Y. Wei, "Deep Feature Flow for Video Recognition," Proceedings - 30th IEEE Conference on Computer Vision and Pattern Recognition, CVPR 2017, vol. 2017-Janua, pp. 41414150, 2017.

[19] K. Kang, H. Li, J. Yan, X. Zeng, B. Yang, T. Xiao, C. Zhang, Z. Wang, R. Wang, X. Wang, and W. Ouyang, "T-CNN: Tubelets with Convolutional Neural Networks for Object Detection from Videos," IEEE Transactions on Circuits and Systems for Video Technology, vol. 28, no. 10, pp. 2896-2907, 2018

[20] W. Liu, S. Liao, and W. Hu, "Perceiving Motion From Dynamic Memory for Vehicle Detection in Surveillance Videos," IEEE Transactions on Circuits and Systems for Video Technology, vol. 29, no. 12, pp. 35583567,2019

[21] G. Montavon, S. Lapuschkin, A. Binder, W. Samek, and K.-R. Müller, "Explaining Nonlinear Classification Decisions With Deep Taylor Decomposition," Pattern Recognition, vol. 65, pp. 211-222, 2017. [Online]. Available: http://www.sciencedirect.com/science/article/ pii/S0031320316303582

[22] K. Simonyan, A. Vedaldi, and A. Zisserman, "Deep Inside Convolutional Networks: Visualising Image Classification Models and Saliency Maps," in 2nd International Conference on Learning Representations, ICLR 2014 - Workshop Track Proceedings, 2014.

[23] D. Balduzzi, B. McWilliams, and T. Butler-Yeoman, "Neural Taylor Approximations: Convergence and Exploration in Rectifier Networks," in ICML, 2016. [Online]. Available: http://arxiv.org/abs/1611.02345

[24] G.-H. Lee, D. Alvarez-Melis, and T. S. Jaakkola, "Towards Robust, Locally Linear Deep Networks," in ICLR, 2019, pp. 1-21.

[25] M. Raghu, B. Poole, J. Kleinberg, S. Ganguli, and J. SohlDickstein, "On the Expressive Power of Deep Neural Networks," in Proceedings of the 34th International Conference on Machine Learning, D. Precup and Y. W. Teh, Eds. International Convention Centre, Sydney, Australia: PMLR, 2017, pp. 2847-2854. [Online]. Available: http://proceedings.mlr.press/v70/raghu17a.html

[26] M. Fischetti and J. Jo, "Deep Neural Networks as 0-1 Mixed Integer Linear Programs: A Feasibility Study," Department of Information Engineering, University of Padova, Tech. Rep., 2017. [Online]. Available: http://arxiv.org/abs/1712.06174

[27] G. Montúfar, R. Pascanu, K. Cho, and Y. Bengio, "On the Number of Linear Regions of Deep Neural Networks," in NIPS, 2014, pp. 1-9. [Online]. Available: http://arxiv.org/abs/1402.1869

[28] T. Serra, C. Tjandraatmadja, and S. Ramalingam, "Bounding and Counting Linear Regions of Deep Neural Networks," 35th International Conference on Machine Learning, ICML 2018, vol. 10, pp. 7243-7261, 2018.

[29] C. H. Cheng, G. Nührenberg, and H. Ruess, "Maximum resilience of artificial neural networks," Lecture Notes in Computer Science, vol. 10482 LNCS, pp. 251-268, 2017. [Online]. Available: https: //link.springer.com/chapter/10.1007/978-3-319-68167-2_18

[30] L. Weng, H. Zhang, H. Chen, Z. Song, C.-J. Hsieh, L. Daniel, D. Boning, and I. Dhillon, "Towards Fast Computation of Certified Robustness for ReLU Networks," in Proceedings of the 35th International Conference on Machine Learning, ser. Proceedings of Machine Learning Research, J. Dy and A. Krause, Eds., vol. 80 Stockholmsmässan, Stockholm Sweden: PMLR, 2018, pp. 5276-5285. [Online]. Available: http://proceedings.mlr.press/v80/weng18a.html

[31] S. Chetlur, C. Woolley, P. Vandermersch, J. Cohen, J. Tran, B. Catanzaro, and E. Shelhamer, "cuDNN: Efficient Primitives for Deep Learning," NVIDIA, Santa Clara, CA 95050, Tech. Rep., 2014. [Online]. Available: https://arxiv.org/pdf/1410.0759.pdf

[32] O. Ronneberger, P. Fischer, and T. Brox, "U-Net: Convolutional Networks for Biomedical Image Segmentation," in Medical Image Computing and Computer-Assisted Intervention, N. Navab, J. Hornegger, W. M. Wells, and A. F. Frangi, Eds. Cham: Springer International Publishing, 2015, pp. 234-241.

[33] Y. LeCun, L. Bottou, Y. Bengio, and P. Haffner, "Gradient-Based Learning Applied to Document Recognition," in Proceedings of the IEEE, vol. 86. IEEE, 1998, pp. 2278-2323.

[34] E. Shelhamer, J. Long, and T. Darrell, "Fully Convolutional Networks for Semantic Segmentation," IEEE Trans. Pattern Anal. Mach. Intell., vol. 39 , no. 4, pp. 640-651, Apr. 2017.

[35] Z. Cao, T. Simon, S. E. Wei, and Y. Sheikh, "Realtime Multi-Person 2D Pose Estimation Using Part Affinity Fields," Proceedings - 30th IEEE Conference on Computer Vision and Pattern Recognition, CVPR 2017, vol. 2017-Janua, pp. 1302-1310, 2017.

[36] D. Balduzzi, M. Frean, L. Leary, J. Lewis, K. W.-D. Ma, and B. McWilliams, "The Shattered Gradients Problem: If Resnets Are the Answer, Then What Is the Question?" in ICML, 2017. [Online]. Available: http://arxiv.org/abs/1702.08591

[37] S. Jastrzebski, D. Arpit, N. Ballas, V. Verma, T. Che, and Y. Bengio, "Residual Connections Encourage Iterative Inference," 6th International Conference on Learning Representations, ICLR 2018 - Conference Track Proceedings, no. 2016, pp. 1-14, 2018.

[38] T. Pfister, K. Simonyan, J. Charles, and A. Zisserman, "Deep Convolutional Neural Networks for Efficient Pose Estimation in Gesture Videos," Lecture Notes in Computer Science, vol. 9003, pp. 538-552, 2015.

[39] P. Chao, C.-Y. Kao, Y.-S. Ruan, C.-H. Huang, and Y.-L. Lin, "Hardnet: A Low Memory Traffic Network," in Proceedings of the IEEE International Conference on Computer Vision, 2019, pp. 3552-3561.

[40] M. Siam, M. Gamal, M. Abdel-Razek, S. Yogamani, M. Jagersand, and H. Zhang, "A Comparative Study of Real-Time Semantic Segmentation for Autonomous Driving," in Proceedings of the IEEE Conference on Computer Vision and Pattern Recognition workshops, 2018, pp. 587597.

[41] S. Rifai, P. Vincent, X. Muller, X. Glorot, and Y. Bengio, "Contractive Auto-Encoders: Explicit Invariance During Feature Extraction," in Proceedings of the 28th International Conference on Machine Learning, ICML 2011, 2011, pp. 833-840.

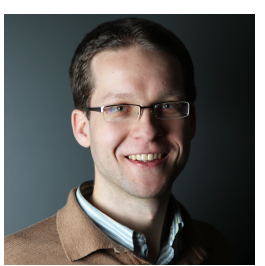

Bodo Rueckauer received the B.Sc. and M.Sc. degrees in physics from ETH Zurich, Zurich, Switzerland, in 2014 and 2016, respectively, and the Ph.D. degree in computational neuroscience from the University of Zurich in 2020 . He was a consultant with Huawei Technologies, and a Research Intern with Intel Labs, OR, USA, in 2020 . He is currently a postdoctoral researcher at the Donders Institute for Brain, Cognition and Behaviour at the Radboud University in Nijmegen, the Netherlands. His research interests include event-based processing with deep neural networks as well as continual, local learning.

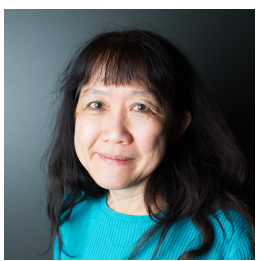

Shih-Chii Liu (M'02 - SM'07) received the PhD degree in Computation and Neural Systems from the California Institute of Technology, Pasadena, in 1997. She worked at various companies, including Gould American Microsystems, LSI Logic, and Rockwell International Research Labs. Currently, she is a professor with the Institute of Neuroinformatics at the University of Zurich, Switzerland. Her interests include low-power neuromorphic eventdriven sensor design; bio-inspired and deep learning algorithms and hardware for energy-efficient, realtime, adaptive intelligent systems. 